\title{
A MODEL OF AGGLOMERATE FORMATION DURING BED WETTING IN THE PROCESS OF DISC GRANULATION
}

\author{
Andrzej Obraniak, Tadeusz Gluba* \\ Technical University of Lodz, Faculty of Process and Environmental Engineering, \\ ul. Wólczańska 213, 90-924 Łódź, Poland
}

\begin{abstract}
This paper presents a method for quantitative assessment of the mechanisms of nucleation and granules growth by layering in the process of bed wetting during periodic disc granulation. This study included two initial, consecutive stages of a process with defined time courses. The first phase was a time period, in which only formation of new nuclei took place, while in the second stage simultaneous nucleation and growth of granules as a result of sticking raw material grains to preexisting nuclei occurred. Different kinds of binding liquid were used for bed wetting in each phase. In the first phase, an aqueous solution of dye was used, and pure distilled water in the second stage. The contribution of particular mechanisms to the formation of agglomerates at different time points within the second phase of the process was determined in this study. To do that the results of bed granulometric analysis, mass balance of size fractions and the analysis of contents of a marker (dye) delivered to the bed with the binding liquid during the first phase in agglomerates were used. To assess the concentration of the dye in different size fractions of the batch, spectrophotometric analysis was utilised. The study was performed using UV-VIS JASCO V-630 spectrophotometer equipped with an integrating sphere. The sieve analysis, spectrophotometric studies and mass balance were used to determine changes in the weight of the dye containing nuclei and of the nuclei containing no dye. The aforementioned analyses were also used to assess changes in the weight of formed granules and of raw material particles attached to nuclei during simultaneous nucleation and growth of granules.
\end{abstract}

Keywords: nucleation, agglomeration, disc granulation, wetting

\section{INTRODUCTION}

In the process of wet granulation, particles subjected to mixing (in tumbling drums, discs, fluidised beds, fast cutting mixers, etc.) are sprayed with a binding liquid which binds the particles together by a combination of capillary and viscous forces. Stronger interstitial bonds may be formed during subsequent processes such as drying and sintering.

To obtain desired properties of produced granules, the designing of both a bed (the choice of finegrained material and binding liquid properties) and process-apparatus parameters (choice of granulator type, parameters of its work, wetting conditions, etc.) are required.

The phenomena and changes occurring at the phase boundary of media involved in the batch movements are of key importance for the course of granulation process and in consequence for the properties of the obtained product.

During the tumbling of a wetted fine-grained bed, interactions between grains of the solid material, droplets of water and air may occur. The size and type of forces interacting between individual material 
grains, or their collections depend strictly on the properties of the media, their mutual affinity and, in particular, on the conditions of bed wetting.

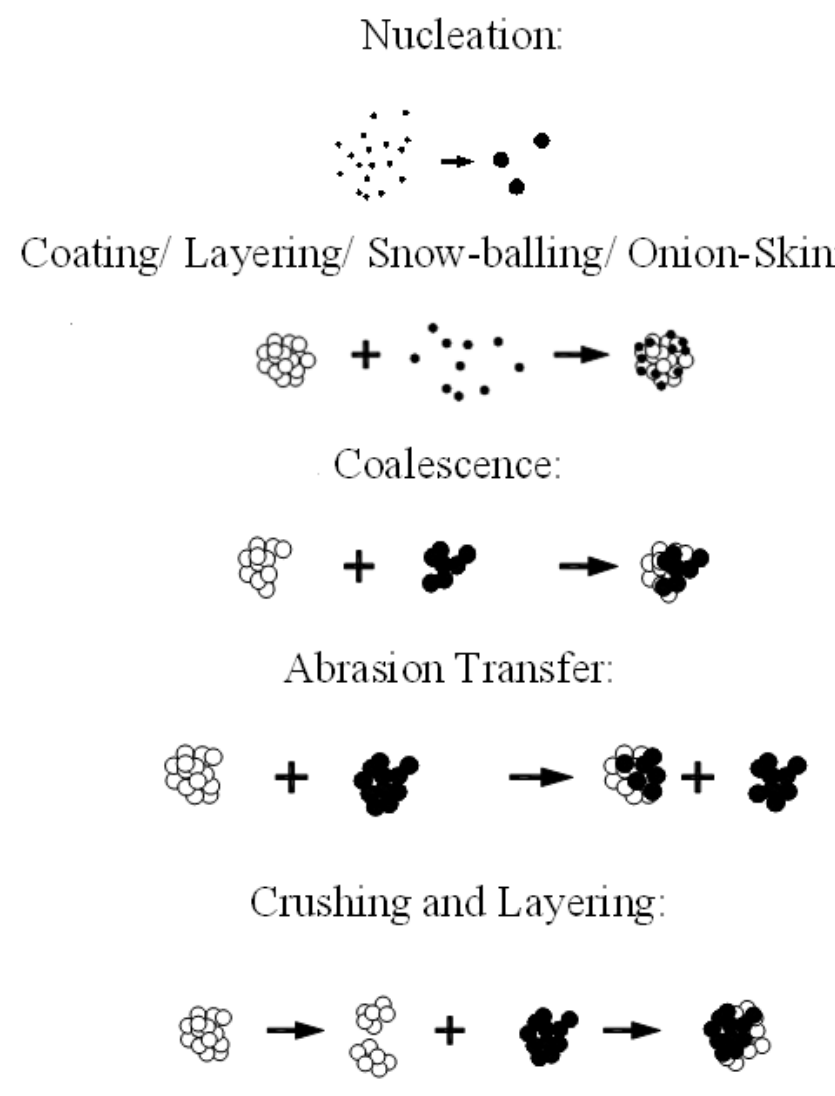

Fig. 1. Mechanisms of agglomerate growth (Sastry and Fuerstenau, 1973)

One of the significant problems to be solved during the wet granulation process is the determination of the conditions of binding liquid supply to the bed of powder material. These conditions determine both the phenomenon of nucleation and also the further growth of agglomerates. Both physical properties of the added binding liquid, its quantity and the method of supply are here of key importance.

Granules nucleation and growth are a complex process which combines several coexisting mechanisms. Sastry and Fuerstenau (1971) systematised these mechanisms and confirmed their existence in their studies. The mechanisms proposed by them are shown in Figure 1.

Recently, an opinion has prevailed in the literature (Ennis and Litster, 1997; Mort, 2009; Tardos et al., 1997), that the process of wet granulation depends on the combination of only three types of mechanisms presented in Figure 2:

- wetting and nucleation, when a liquid binder is in contact with dry material and spreads in the bed, thus producing the nuclei of granulation,

- consolidation and growth, when collisions between particular granules, granules and the bed, or granules and walls of the device, cause consolidation and growth of the granules;

- attrition and breakage, when due to collisions, portions of material are exchanged and weak granules are disintegrated.

Bed wetting and subsequent nucleation constitute a very important stage that determines the further run of the granulation process, and consequently, exerts an influence on the product properties.

Wetting and nucleation are processes of bringing binding liquid into contact with the dry powder and simultaneous liquid distribution inside the powder. This important stage in granulation is rarely 
identified and separated from other effects such as layering, coalescence, and attrition, and thus the knowledge about the course and the control of the nucleation process is limited. The nucleation zone, or the wetting zone, are defined as an area where the binding liquid and the surface of powder particles interact with each other and form pre-nuclei (Schaafsma et al., 1999). The size distribution of these initial nuclei depends on the processes occurring in the nucleation zone, although some other processes, such as mechanical agitation, which take place in the granulator, can then affect this distribution (Iveson et al., 2001).

a) wetting and nucleation

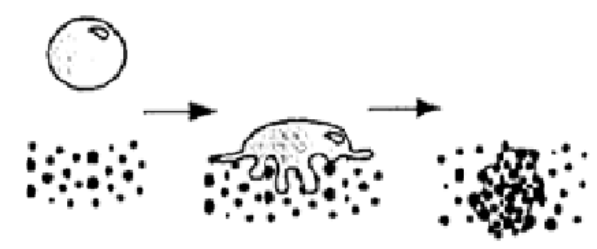

b) consolidation and growth

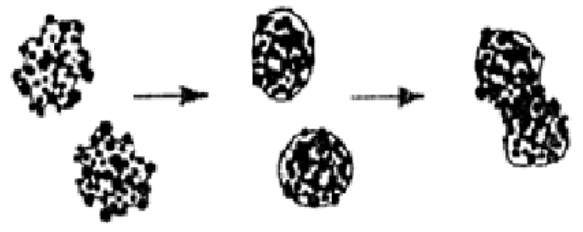

c) attrition and breakage

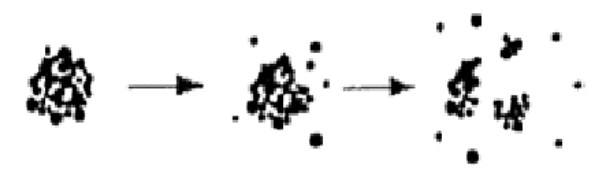

Fig. 2. Mechanisms of agglomerate growth, modern approach (Ennis and Litster, 1997)

Schaafsma et al. (1999) and Iveson et al. (2001) found that during wetting and nucleation, two processes are of most importance:

- nuclei formation which is a function of thermodynamics and kinetics of wetting,

- dispersion of a binding agent (efficiency of powder and liquid mixing), which is a function of process variables.

During wetting and nucleation liquid penetrates powder pores, forms nuclei and then starts migrating outwards, when the nuclei grow.

Schaafsma et al. (1998) and Butensky and Hyman (1971) stated that the time of liquid penetration in the material depended both on the wetting thermodynamics and kinetics, which are dependent on the liquid viscosity and effective pore dimensions in the bed powder.

An important wetting parameter that affects nucleation conditions is also the degree of liquid binder disintegration. It depends strictly on the method of binding water supply and has an effect on the powder-liquid mixture quality.

Gluba (2003) reported that binding liquid dispersion had an effect on particle size distribution of the granulated product. The effect of liquid binder dispersion on the mechanisms of nucleation and granule growth in fluid bed melt agglomeration was confirmed by Abberger et al. (2002).

For melt pelletisation in a high shear mixer Schæfer and Mathiesen (1996) proposed two different nucleation mechanisms, depending on the relative size of droplets and particles. 
If a drop is large in comparison with the size of powder grain, nucleation occurs by immersion of small grains into large drops. This produces nuclei with saturated pores.

Nucleation with relatively small drops occurs by distribution of drops on the surface of grains which will then start to coalesce. This will facilitate the formation of nuclei which may have air trapped inside.

In case of immersion, the binding liquid contacts one time with the powder and penetrates into the capillary pores, resulting in the formation of initial agglomerates which are highly saturated with liquid. Kinetic models for granule nucleation by the immersion mechanism were presented by Hounslow et al. (2008).

The dispersion of binder in the granular material depends on the effectiveness of both powder and liquid mixing which is, in turn, related to the type of the used device and its operating parameters.

At the time of binding liquid delivery to the tumbling fine-grained bed, separate or combined processes of nucleation and agglomerates growth may occur at the various stages of the process. The ability to distinguish between different mechanisms would allow for more accurate understanding of phenomena taking place in the granulated bed.

The study attempts to separate the mechanisms of nucleation and mechanisms of granule growth by layering and to determine their participation in the formation of agglomerates in various periods of bed wetting in the process of disc granulation.

\section{AIM}

The aim of this study was to develop and verify methods for quantitative evaluation of the mechanisms of nucleation and layering in the process of disc granulation, utilising spectrophotometric analysis of the content of marker (dye), supplied to the bed with the binding liquid, in the agglomerates.

\section{PRELIMINARY STUDIES}

During the preliminary studies, basic parameters of a periodic disc granulation process such as: rotational speed and angle of the disc inclination, weight of fine-grained load, as well as the position and intensity of binding liquid drops delivery, were selected. The determined parameters guaranteed that in the initial period, droplets falling on the tumbling bed were in contact only with fine-grained material to form subsequent individual nuclei (every droplet formed one nucleus). This phase of the process, associated only with the undisturbed formation of granules nuclei, can be described as the phase of "pure nucleation". Nuclei formed in this period are surrounded by loose fine-grained material and remain dry on the surface. The duration of this phase was selected in such a way as to exclude the possibility of drop hitting on pre-formed nucleus.

It was found that the delivery of liquid after this period can lead to both the formation of subsequent nuclei and the growth of previously formed nuclei by the attachment of grains of dry material. The attachment of grains to the nucleus (layering) is possible when a droplet of liquid falls on a nucleus and leads to an increase in its moisture. Such a nucleus is, for some time, wet on the surface, which facilitates the attachment of adjacent powder grains owing to capillary and surface tension forces. The probability of such nuclei enlargement increases with increasing the time of liquid delivery, and thus with the amount of formed nuclei. The phase of simultaneous nucleation and layering continues until 
the first granules formed by coalescence appear in the bed. This means that nuclei and granules which are wet on the surface begin to collide and join each other to form larger agglomerates.

The range of this research work was limited to the first two phases of the process, during which only pure nucleation or nucleation with layering occur, but particles coalescence is not observed yet.

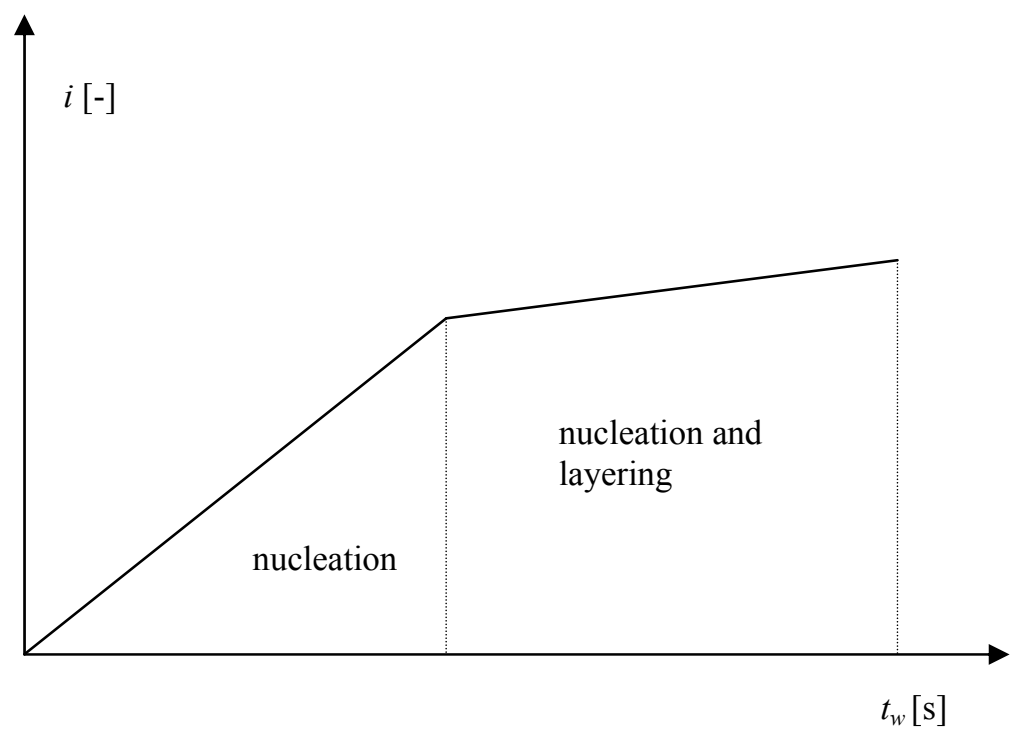

Fig. 3. The increase of agglomerates number during the delivery of binding liquid to the fine-grained bed

It was found that during these phases the mechanisms of disintegration (crushing, abrasion) of previously formed agglomerates do not occur, because particles are "suspended" in non-granulated loose material and circulate together with it, so their contact with each other and with the walls of the apparatus is negligible.

An increase in the amount of agglomerates during the delivery of the binding liquid to the fine-grained bed is presented schematically as a 2-stage process in Figure 3.

\section{THE STUDY APPARATUS AND RESEARCH RANGE}

The investigations of nucleation and granule growth were carried out on an experimental setup shown schematically in Figure 4.

Granulating disc (1) of a diameter $D=0.5 \mathrm{~m}$ and a height of the rim $h_{T}=0.1 \mathrm{~m}$ driven by an electric engine with gear (2) was the key element of the apparatus. The disc rotational speed was established by an inverter (3). The binding liquid was delivered from the tank (4) to a fine-grained bed tumbling in the disc by means of a hydraulic sprinkler (5) tipped with a nozzle that generated droplets of ca. $2.5 \mathrm{~mm}$ in size. The use of one nozzle provided the delivery of liquid drops to a specific place in the tumbling bed and thus in the first phase of the process drops fell only on the loose material. A constant flow of the binding liquid measured with the use of a rotameter (6) was applied. Drops of the binding liquid were delivered for a given period of time that closely depended on the current stage of research. On the basis of preliminary studies it was found that when the established intensity of drops delivery was applied, the phase of pure nucleation lasted for $\tau=20 \mathrm{~min}$, and the stage of nucleation and layering for the next $17 \mathrm{~min}$. When this time was exceeded, first signs of the coalescence of particles in the processed bed had been observed. 


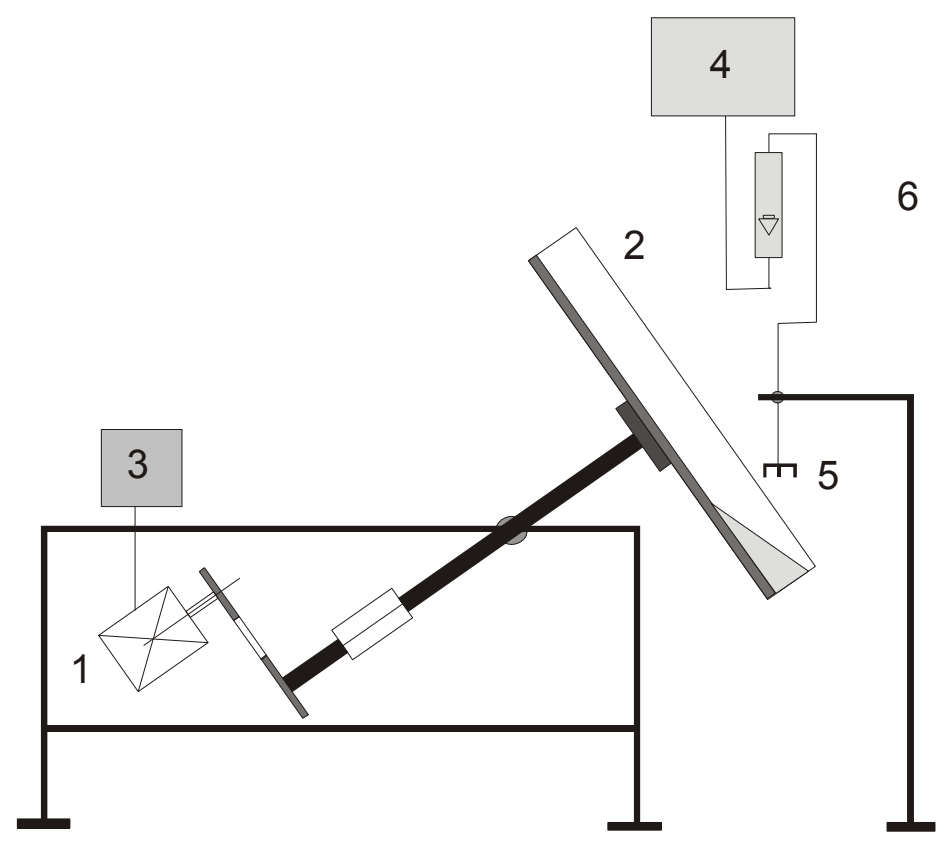

Fig. 4. Schematic diagram of the equipment,

1 - electric engine, 2 - granulating disc, 3 - inverter, 4 - tank, 5 - hydraulic sprinkler, 6 - rotameter

The following process and equipment parameters were used in this study:

- disc rotational speed $n=10 \mathrm{rpm}$,

- the angle between the disk axis and level $\alpha=40^{\circ}$,

- the mass of fine-grained load (raw material) $m_{s}=1.0 \mathrm{~kg}$,

- the intensity of the binding liquid delivery $Q_{w}=8.75 \mathrm{dm} 3 / \mathrm{min}$.

- the time of liquid delivery from $t=20$ up to 37 minutes.

Fine-grained foundry bentonite (raw material) was used as the study material and $0.1 \%$ aqueous solution of dye (black) and pure distilled water were used as the binding liquid. The selected dye was characterised by a very high intensity of material staining, even at its low concentrations in water. Such a low addition of the dye did not cause any changes of physical and chemical properties of water.

\section{MATERIALS AND METHODS}

A predetermined mass of material placed in the granulator was wetted with droplets during disc rotation. Every attempt of granulation consisted of two successive stages, in which different kind of the binding liquid was delivered.

In the first stage - corresponding to the pure nucleation phase - an aqueous solution of the dye delivered for a period of $\tau=20 \mathrm{~min}$ was used as the liquid binder. In the next stage - nucleation and layering - pure distilled water used as the binding liquid was delivered for time $t$, equal to $t=10,14$ and $17 \mathrm{~min}$ in subsequent trials. In order to assess the properties of the batch obtained after a specified period of the binding liquid delivery, the entire batch was weighed, separated into size fractions using a set of sieves with mesh sizes: 0.2, 1.0, 2.0, 3.0, 4.0, 5.0, 6.3, $8 \mathrm{~mm}$, and then dried in a drying oven at $90^{\circ} \mathrm{C}$ for $24 \mathrm{~h}$. After drying, the weight of every size fraction was determined on an analytical balance and on this basis granulometric composition of the batch was estimated.

It was observed that all agglomerates obtained after $20 \mathrm{~min}$ of liquid delivery (during pure nucleation phase) were in the size class $d=2$ to $4 \mathrm{~mm}$. Therefore, it was assumed that nuclei obtained in the second phase would have the same size and that agglomerates larger than $4 \mathrm{~mm}$ are granules formed as a result 
of nuclei layering. In order to analyse the mechanisms of agglomerate formation and growth, the content of the dye in the selected size fractions of batch was compared for different phases of the process. For the analysis of the content of the dye, the whole feed was divided into four size fractions:

- non-granulated material $(d<0.4 \mathrm{~mm})$

- pieces resulting from the destruction $(d=0.4 \div 2 \mathrm{~mm})$

- $\operatorname{nuclei}(d=2 \div 4 \mathrm{~mm})$

- $\operatorname{granules}(d>4 \mathrm{~mm})$.

The dye content in the aforementioned fractions was measured spectrophotometrically. The study was performed on a UV-VIS JASCO V-630 spectrophotometer which is designed for the measurement of the absorption spectra in the range from 190 up to $1100 \mathrm{~nm}$. The so- called integrating sphere was used to measure radiation reflected from the surface of the stained powder material. To assess the content of the dye in the sample the following two parameters were used: absorbance and transmittance. At the beginning, a scaling curve was drawn on the basis of the measurements of the aforementioned parameters in samples with the known dye content. A sample with the known maximum dye content was prepared from nuclei containing the entire dye enclosed in the delivered binding liquid, formed during pure nucleation phase. The concentration of the dye in nuclei $c_{0}$ was calculated by dividing the weight of the dye delivered with the liquid by the weight of formed nuclei. The whole mass of the formed nuclei was crumbled into powder in a dry state and homogenised in the entire volume. Subsequent samples with lower dye concentrations $(c)$ were obtained by mixing, in known mass proportions, the stained powder from nuclei with the fresh raw material used during the study. The obtained scaling curve (baseline) is shown in Figure 5.

Using the scaling curve the concentration of the dye in the batch fractions obtained at the stage of nucleation and layering $(c)$ was determined on the basis of the values of absorbance and transmittance measured for the averaged powder samples derived from each of the four size fractions.

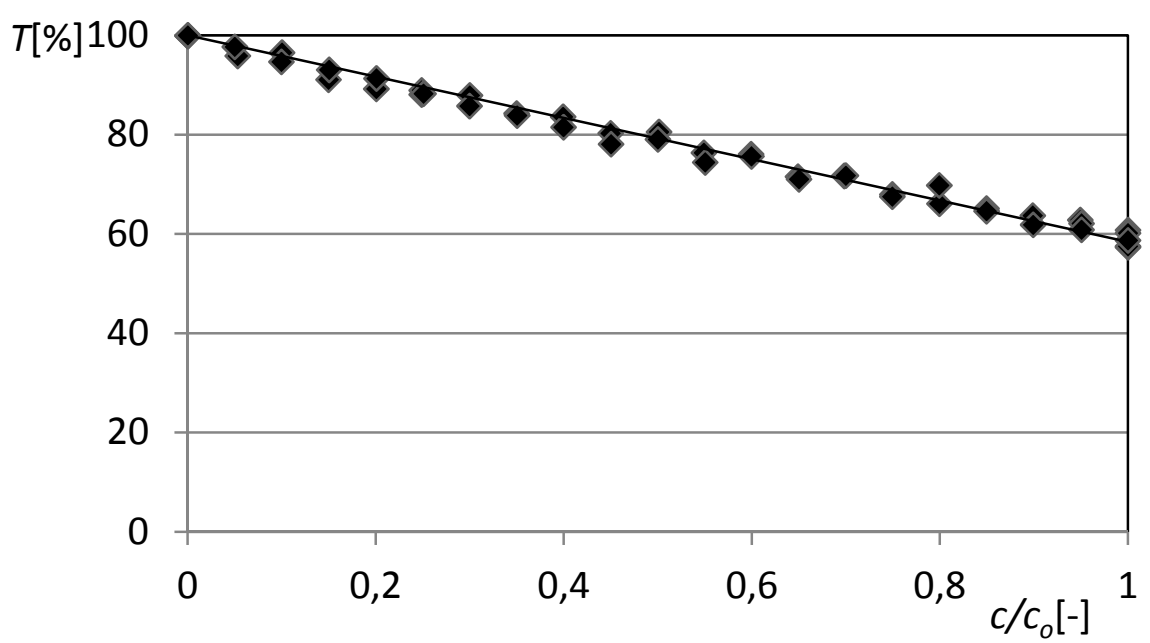

Fig. 5. The baseline for the determination of dye concentration in samples

\section{RESULTS}

Based on the results of sieve analysis, the mass balance of the processed bed was conducted for both phases of granulation. A diagram of the aforementioned balance for the dried material is shown in Figure 6 . 


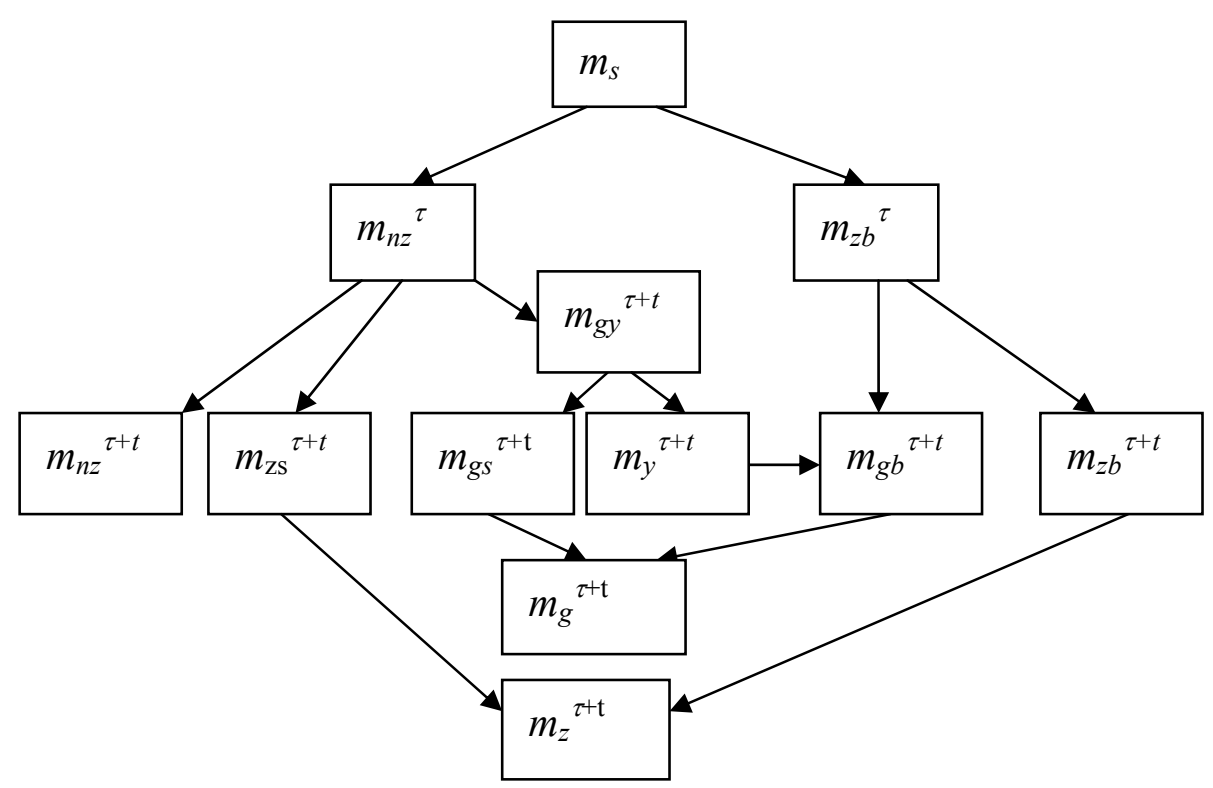

Fig. 6. Mass balance in the processed bed

Due to the fact that the mass of the dye delivered with the liquid to $1 \mathrm{~kg}$ of raw material was only $0.175 \mathrm{~g}$, it was regarded as negligible in the considerations on the balance of mass (after drying). However, the content of the dye was fully sufficient for the purposes of spectrophotometric analysis.

According to the results of the performed sieve analysis, after the end of delivery of the binding liquid with the dye (for $\tau=20 \mathrm{~min}$ ) only the following two batch fractions were obtained: nuclei, in which the entire binding liquid and the dye were accumulated $(d=2 \div 4 \mathrm{~mm})$, and unprocessed raw material $(d<0.4 \mathrm{~mm})$.

After drying, the weights of these fractions were $m_{z b}{ }^{\tau}$ and $m_{n z}{ }^{\tau}$, respectively, and they satisfy Equation (1):

$$
m_{s}=m_{n z}^{\tau}+m_{z b}^{\tau}
$$

During further bed granulation, in the second phase, that was initiated by the delivery (for a definite time) of pure water droplets to the bed tumbling in the disc, there is a possibility of the formation of new nuclei (not containing dye), and generation of granules as a result of incorporation of raw material grains into nuclei formed both in the first and the second stage of the process. There is also a possibility that some fragments of nuclei and granules (pieces) may be formed as a result of destructive interactions in the tumbling bed (fraction $d=0.4 \div 2 \mathrm{~mm}$ ). On the basis of sieve analysis, it was concluded that such a fraction did not appear in the studied two stages of the process and therefore it was not taken into account in the analysis of mass balance.

Figure 7 presents the mass distribution of individual grain fractions of the bed after different times of the binding liquid delivery. In the second phase, with an increase in time of the binding liquid delivery, a growing mass share of the fraction of the nuclei could be observed, even though some of them increased their size after the attachment of the raw material grains and were transferred into the fraction of granules. This still demonstrates a high rate of nucleation at this stage of the process. 


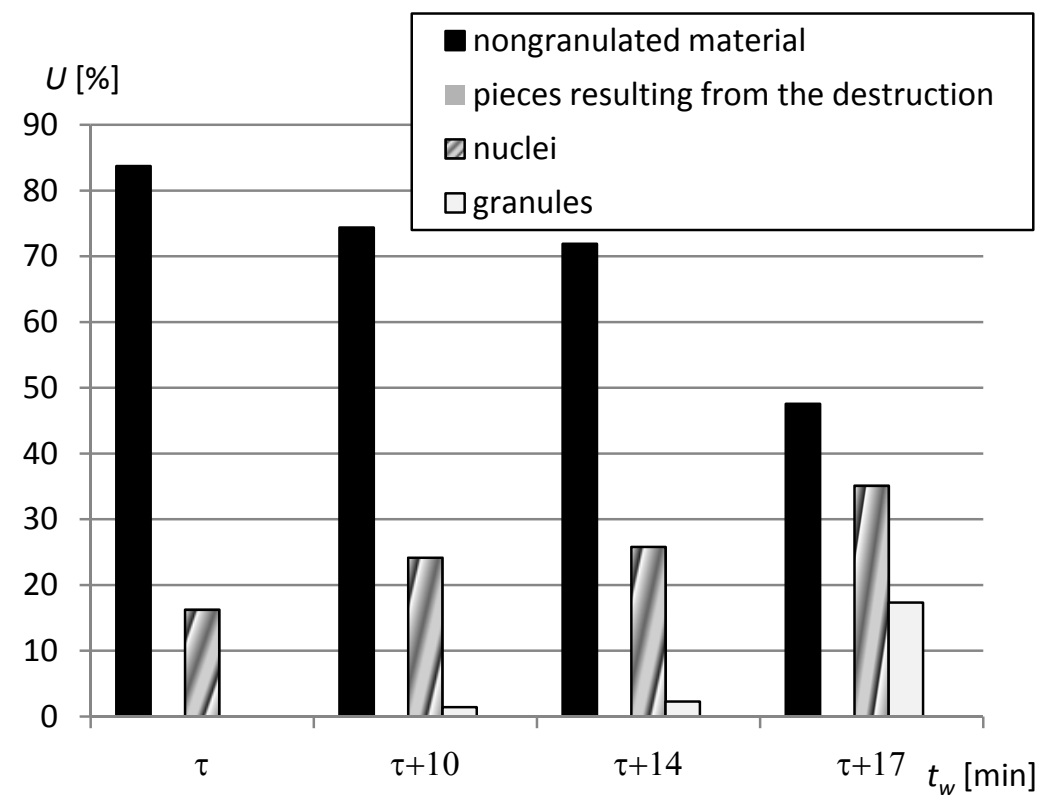

Fig. 7. The comparison of mass distribution of bed fractions at different times of the bed wetting

The mass balance for individual batch fractions after their wetting with water for $t$ time in the second stage of the process is described Equations (2) to (5):

$$
\begin{gathered}
m_{z b}^{\tau}=m_{z b}^{\tau+t}+m_{g b}^{\tau+t}-m_{y}^{\tau+t} \\
m_{n z}^{\tau}=m_{n z}^{\tau+t}+m_{g y}^{\tau+t}+m_{z s}^{\tau+t}=m_{n z}^{\tau+t}+m_{g s}^{\tau+t}+m_{y}^{\tau+t}+m_{z s}^{\tau+t} \\
m_{z}^{\tau+t}=m_{z b}^{\tau+t}+m_{z s}^{\tau+t} \\
m_{g}^{\tau+t}=m_{g s}^{\tau+t}+m_{g b}^{\tau+t}
\end{gathered}
$$

An analysis of the dye content in the individual batch size fractions in the second stage, after a defined time $t$ of pure water delivery, suggests possible mechanisms that occur in the bed at this stage of the process.

The set of equations necessary for the determination of nuclei masses, which were formed during the second stage of the process or turned into granules at that time, as well as those which retained their size, requires the addition of equation describing the dye concentration in the separate size fractions (Equations $6 \div 8$ ).

$$
\begin{gathered}
c_{0}=\frac{m_{B}}{m_{z b}^{\tau}}=\frac{m_{B}^{\tau+t}(z)}{m_{z b}^{\tau+t}} \\
c_{z}^{\tau+t}=\frac{m_{B}^{\tau+t}(z)}{m_{z}^{\tau+t}} \\
c_{g}^{\tau+t}=\frac{m_{B}^{\tau+t}(g)}{m_{g}^{\tau+t}}
\end{gathered}
$$

The dye concentration in a given fraction was calculated from the values of transmittance obtained during the spectrophotometric analysis of nuclei containing a known dye concentration, and formed in the first stage of the process. 
Changes in the dye concentration in two batch fractions: nuclei and granules during the second stage of the process are shown in Figure 8.

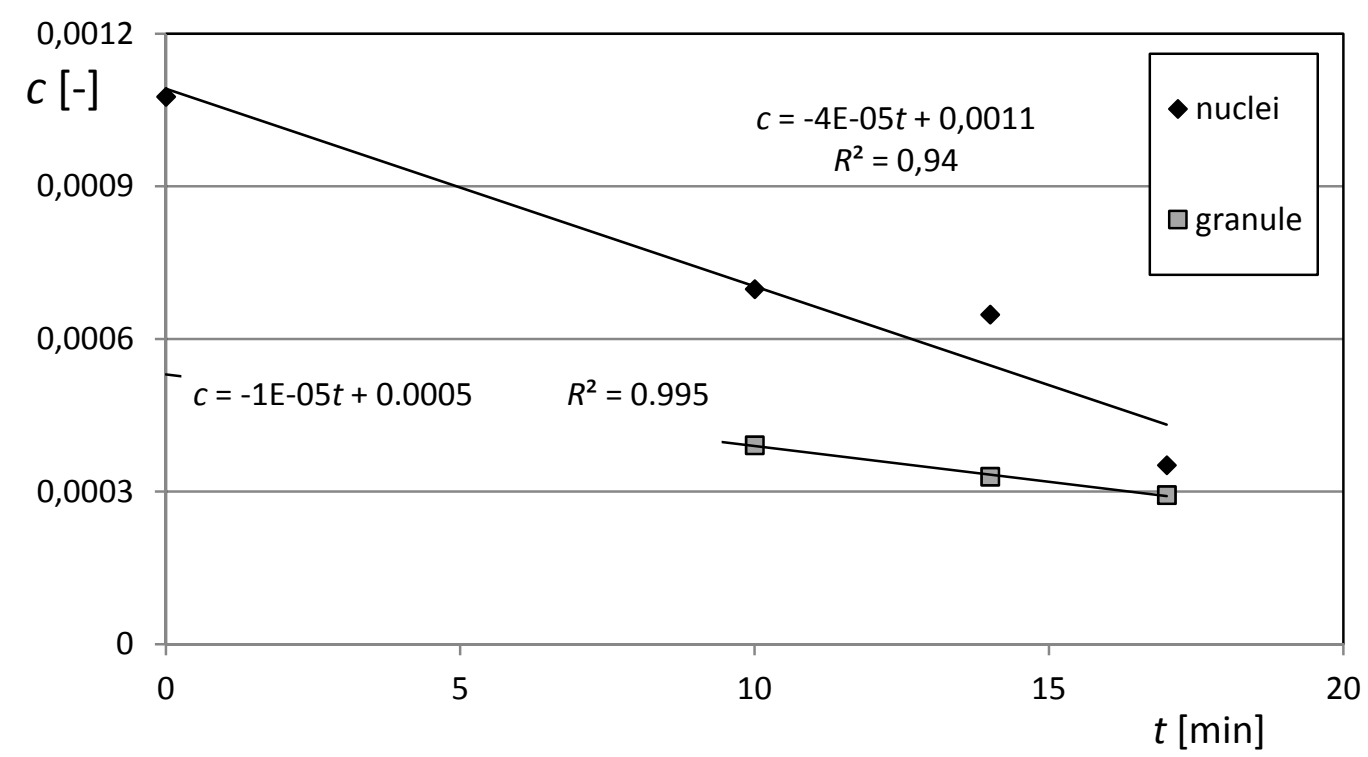

Fig. 8. Changes of dye concentrations in the fractions of nuclei and granules in the second stage of the process

Based on the observations, it can be concluded that with an increasing time of liquid delivery, the dye concentration in both fractions decreases. A decline in the dye concentration in the nuclei fraction is associated with two mechanisms: the formation of new nuclei that contain no dye and the transfer of dye-containing nuclei to the fraction of granules as a result of raw material grains attachment (layering). On the other hand, a decrease of the dye concentration in the granules fraction is associated with both an increase in their size by attaching grains of raw material and the formation of granules from nuclei that contain no dye.

On the basis of dye content in the fractions of nuclei or granules at a particular time within the second phase of the process (calculated from Equations $6 \div 8$ ), the ratio between the mass of nuclei formed in the first phase and those formed during wetting with water, after a time $\tau+t$ could be calculated using Equation (9).

$$
\frac{m_{z b}^{\tau+t}}{m_{z}^{\tau+t}}=\frac{c_{z}^{\tau+t}}{c_{0}}
$$

On the basis of the proposed equations, resulting from the sieve analysis, spectrophotometric measurements and mass balance, changes in the weight of dye-containing nuclei and those without dye as well as in the weight of the formed granules and raw material attached to nuclei at various stages of the process could be determined. The changes in the mass fractions of aforementioned parts of the bed in time are shown in Figure 9.

According to this study, the mechanism of nucleation of new nuclei (not containing the dye) dominates in the first stage of the second phase (up to 34 min of process). A slight increase was observed in the weight of nuclei with the dye, which due to layering were transferred to the size class of granules, and in the weight of raw material attached to nuclei. A significant increase in the weight of granules fraction was observed only at the end of this stage (after $34 \mathrm{~min}$ of the process), which indicates that the layering mechanism becomes more significant at that time. This is associated with a greater probability 
of additional wetting of the increasing number of formed nuclei, allowing for the attachment of new grains of the material.

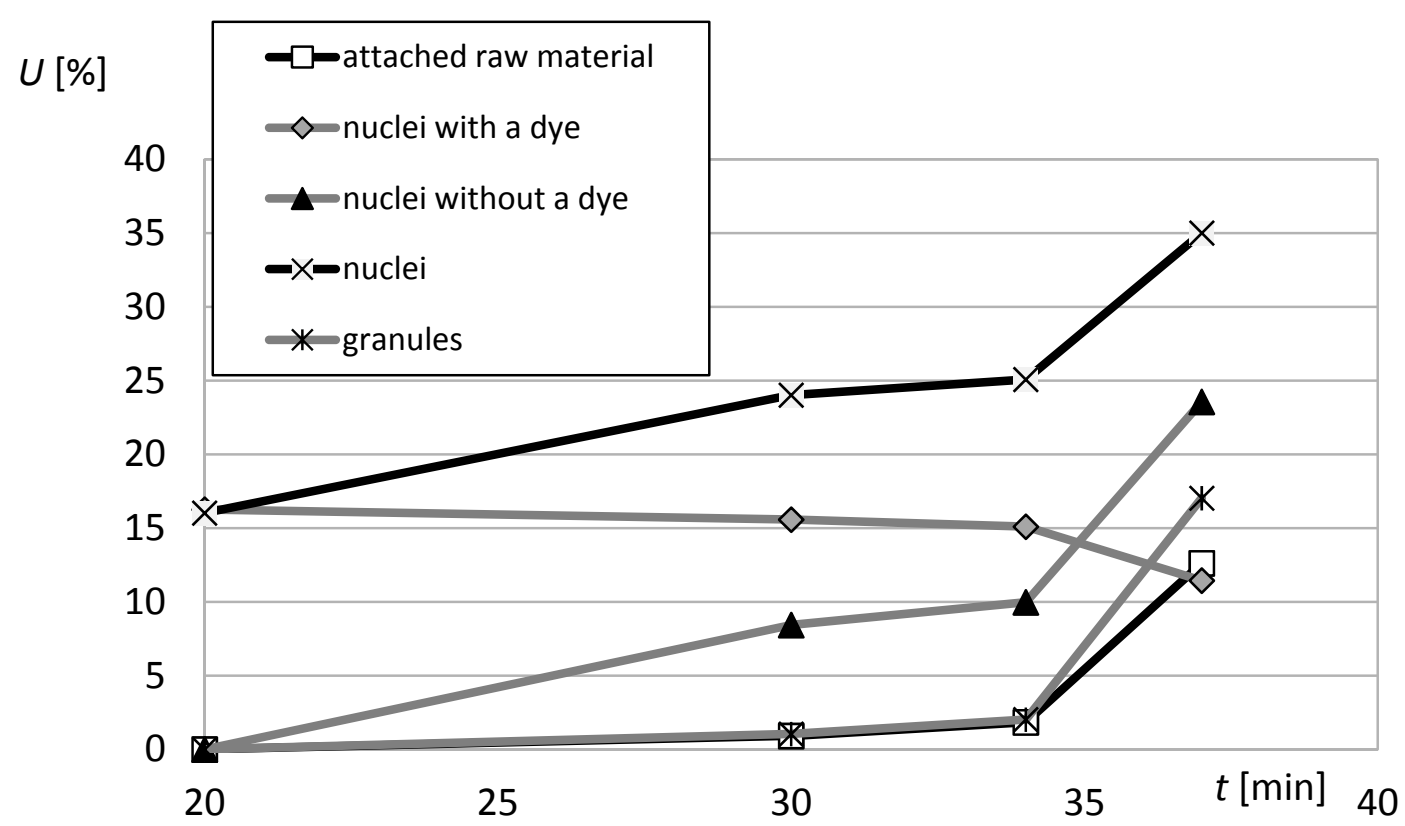

Fig. 9. Changes of dye concentrations in the fractions of nuclei and granules in the second stage of the process

\section{CONCLUSIONS}

The study of granulation at the stage of bed wetting demonstrated the usefulness of the proposed method utilising granulometric analysis, mass balance of batch and the measurement of the dye content in the obtained fractions, for the quantitative assessment of agglomerate growth mechanisms.

The solution of the proposed model and the results of grain and spectrophotometric analysis allow for:

- the distinction between nuclei formed at the stage of "pure nucleation" and those formed at the stage of simultaneous nucleation and layering, as well as for the determination of their masses,

- the determination of proportion of nuclei formed in the first stage of the process that increased their size by layering and then turned into granules

- the determination of the mass of raw material, which due to nucleation and layering, transferred in the second stage of the process, to a fraction of granules, and a fraction of nuclei

- the determination of time intervals, in which the mechanisms of formation (nucleation) and agglomerates growth by layering are dominant

The study was carried out under the research project N N209 096135 in the Department of Process Equipment in 2008 - 2011.

\section{SYMBOLS}

$c \quad$ dye concentration in nuclei $\left(c_{z}\right)$ or granules $\left(c_{g}\right)$ at the stage of simultaneous nucleation and layering, the concentration of dye in nuclei after the phase of pure nucleation, - 


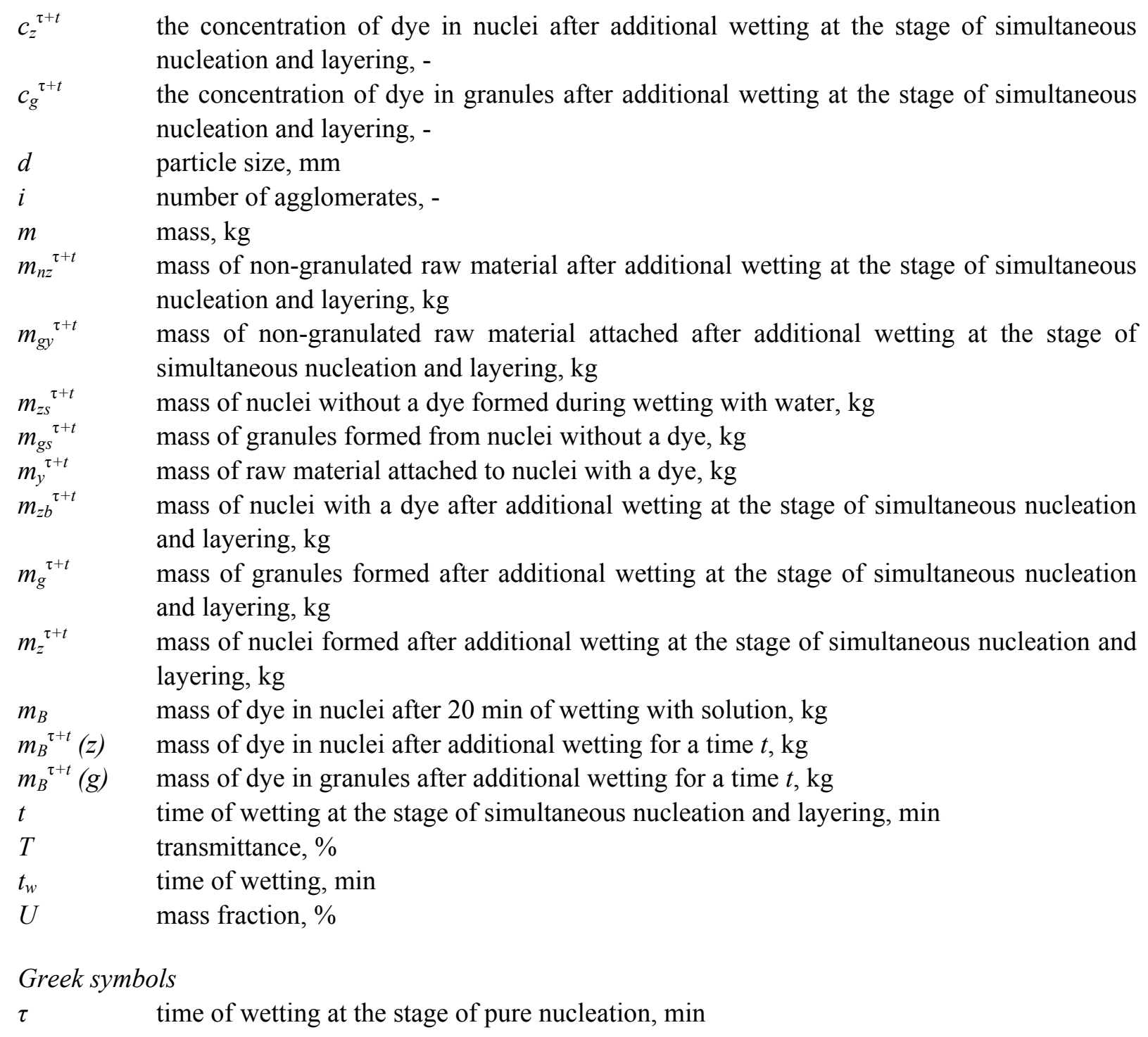

\section{REFERENCES}

Abberger T., Seo A., Schaefer T., 2002. The effect of droplet size and powder particle size on the mechanisms of nucleation and growth in fluid bed melt agglomeration. Int. J. Pharm., 249, 185-197. DOI: 10.1016/S03785173(02)00530-6.

Butensky M., Hyman D., 1971. Rotary drum granulation. An experimental study of the factors affecting granule size. Ind. Eng. Chem. Fundam., 10, 212-219. DOI: 10.1021/i160038a005.

Ennis B.J., Litster J.D., 1997. Particle size enlargement, In: Perry R., Green D. (Eds.), Perry's Chemical Engineers' Handbook. 7th edition, McGraw-Hill, New York, 20.56-20.89.

Gluba T., 2003. The effect of wetting liquid droplet size on the growth of agglomerates during wet drum granulation. Powder Technol., 130, 219-224. DOI: 10.1016/S0032-5910(02)00269-3.

Hounslow M.J., Oullion M., Reynolds G.K., 2008. Kinetic models for granule nucleation by the immersion mechanism. Powder Technol., 189, 177-189. DOI: 10.1016/j.powtec.2008.04.008.

Iveson S.M., Litster J.D., Hapgood K., Ennis B.J., 2001. Nucleation, growth and breakage phenomena in agitated wet granulation processes: A review. Powder Technol., 117, 3-39. DOI:10.1016/S0032-5910(01)00313-8.

Mort P.R., 2009. Scale-up and control of binder agglomeration processes - Flow and stress fields. Powder Technol., 189, 313-317. DOI:10.1016/j.powtec.2008.04.022.

Sastry K.V.S., Fuerstenau D.W., 1973. Mechanisms of agglomerate growth in green pelletization. Powder Technol., 7, 97-106. DOI:10.1016/0032-5910(73)80012-9. 
Schaafsma S.H., Kossen N.W.F., Mos M.T., Blauw L., Hoffman A.C., 1999. Effects and control of humidity and particle mixing in fluid-bed granulation. AIChE J., 45, 1202-1210. DOI: 10.1002/aic.690450606.

Schaafsma S.H., Vonk P., Segers P., Kossen N.W.F., 1998. Description of agglomerate growth. Powder Technol., 97, 183-190. DOI: 10.1016/S0032-5910 (97) 03399-8.

Schaefer T., Mathiesen C., 1996. Melt pelletization in a high shear mixer: IX. Effects of binder particle size. Int. J. Pharm., 139, 139-148. DOI:10.1016/0378-5173(96)04548-6.

Tardos G.I., Irfan-Khan M., Mort P.R., 1997. Critical Parameters and limiting conditions in binder granulation of fine powders. Powder Technol., 94, 245-258. DOI:10.1016/S0032-5910(97)03321-4.

Received 28 September 2011

Received in revised form 28 January 2012

Accepted 28 January 2012 